\title{
4. FACTORS AFFECTING RADAR-METEOR ECHO DURATIONS
}

\author{
A. HAJDUK \\ (Astronomical Institute of the Slovak \\ Academy of Sciences, Bratislava, Czechoslovakia)
}

\begin{abstract}
A BSTRACT
The analysis of two factors influencing radar-meteor echo durations leads to the conclusion, that (1) the echo duration depends considerably on the train position with respect to the sensitivity contours of the radar; (2) the mean echo duration changes with respect to the radiant motion of a meteor shower. As a consequence of the factors mentioned above, the magnitude function, or the mass distribution function depends significantly on the observational conditions, as well as on the choice of the range and time intervals of the investigated sample.
\end{abstract}

The correlation between the visual magnitude and radar echo duration of meteors is very often used for the determination of the magnitude function and properties of showers connected with it. A diagram based on the combined visual-radar observations shows a wide spread. As has been shown by Lindblad (1956) and Millman and McKinley (1956), this scatter is so wide, that a given duration class may extend over eight magnitudes, or a given magnitude class may include durations which vary as much as 1000:1. There are many factors which influence the echo durations, of which two are analysed in the present paper.

\section{The Dependence of Echo Duration on the Train Position with Respect to the Sensitivity Contours of the Radar}

The material used in this treatment comprises about 26000 meteor echoes observed with the Ondřejov meteor radar during the Orionid period in the years 1961-65.

With respect to the sensitivity contours of the radar equipment (Hajduk, 1965a), for larger distances there are recorded relatively brighter meteors than for smaller ones. We can expect, therefore, that for larger distances echoes with relatively longer durations will be obtained. But, as is shown in Figure 1, the mean echo duration, obtained by the sum of the log duration, remains constant along the whole scale of the range. As further analysis shows, there is no change even in percentage of longduration echoes with range. That means that the echoes of the brighter meteors at larger distances have the same duration as the echoes of fainter ones at the smaller distances. In other words, the sensitivity of the apparatus which is different in range affects the duration of echoes. Figure 1 shows the correlation between mean echo 

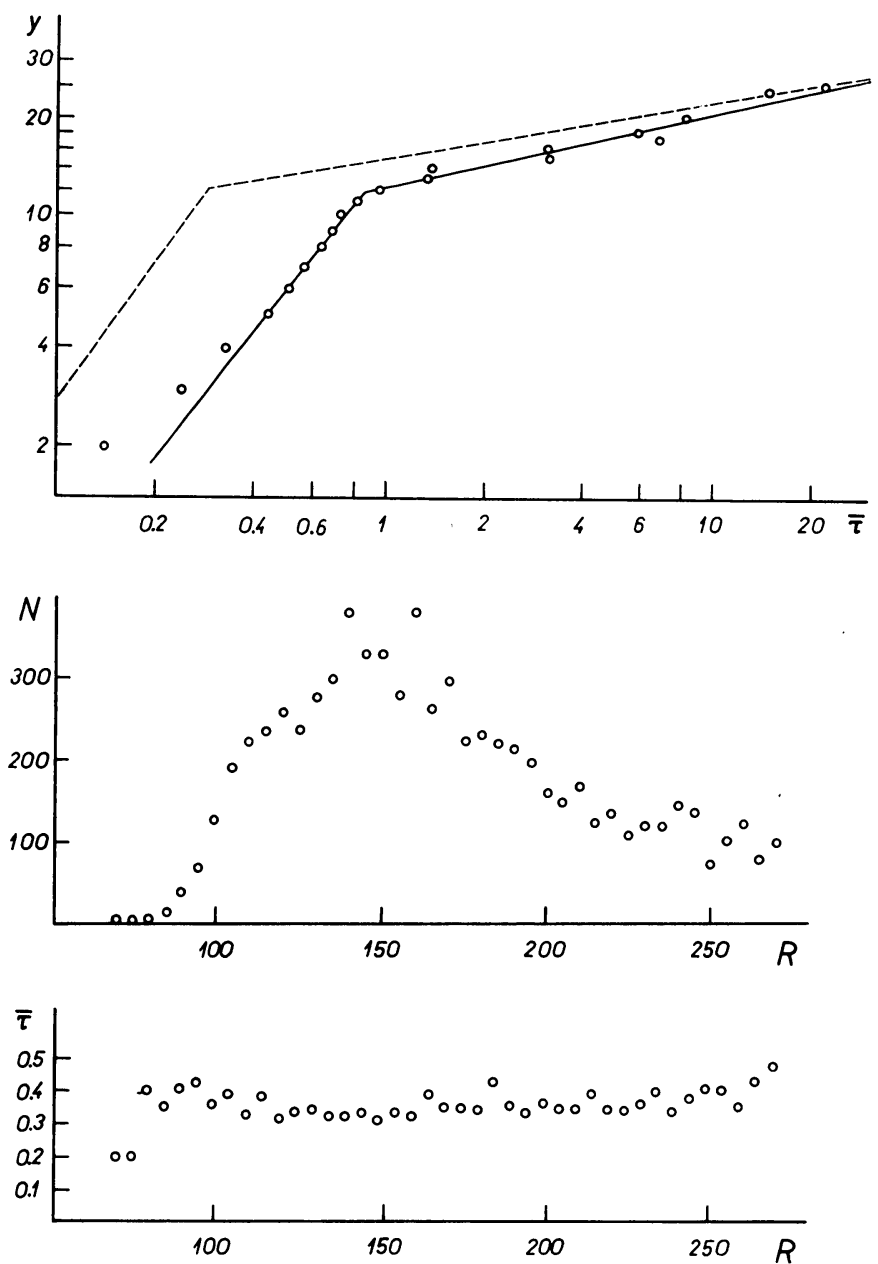

FIG. 1. Above: The correlation of the mean echo duration (in sec) and echo amplitude (relative units). Middle: The range distribution of echoes. Below: The dependence of the mean echo duration (in sec) on range (in $\mathrm{km}$ ).

duration and echo amplitude. The latter is expressed in relative units corresponding to the echo width on the range-time record, since the log echo amplitude-log echo width relation is linear (Hajduk, 1965b). The break of the curve corresponds to the limit point between overdense and underdense trains. The dashed line expresses the correlation corrected for the effect of the image of the echo amplitude on the echo duration. If we project on the horizontal axis the vertical size of the echo image, which corresponds to the amplitude, we obtain the duration which is obviously added to the echo proper duration. The dashed line in Figure 1 was obtained by subtracting this vertical size from all echoes. With this correction the break of the curve is shifted 
along the axis of mean duration, but not in amplitude. The best value of mean duration, corresponding to the critical value of line density, should lie between these two points and should be of the order $0.5 \mathrm{sec}$. In our case this value corresponds to meteors of approximately fifth magnitude and is in good agreement with Millman and McKinley's (1956) and Lindblad's (1956) results.

\section{The Dependence of the Echo Duration on the Position of the Radiant}

The changes in percentage of the long-duration echoes have been studied with respect to dependence on the radiant zenith distance for two showers, Orionids and Geminids. Figure 2 shows the percentage of echoes with the duration of $\tau \geqslant 0.5 \mathrm{sec}$, $\tau \geqslant 1 \mathrm{sec}$, and $\tau \geqslant 5 \mathrm{sec}$, taken over the Orionid period. In comparison with all echoes, beginning at $\tau=0.1 \mathrm{sec}$, considerable changes in the relative number of long-duration echoes are seen. The dependence is nearly symmetrical about the time of radiant culmination corresponding approximately to 5:20 MET.

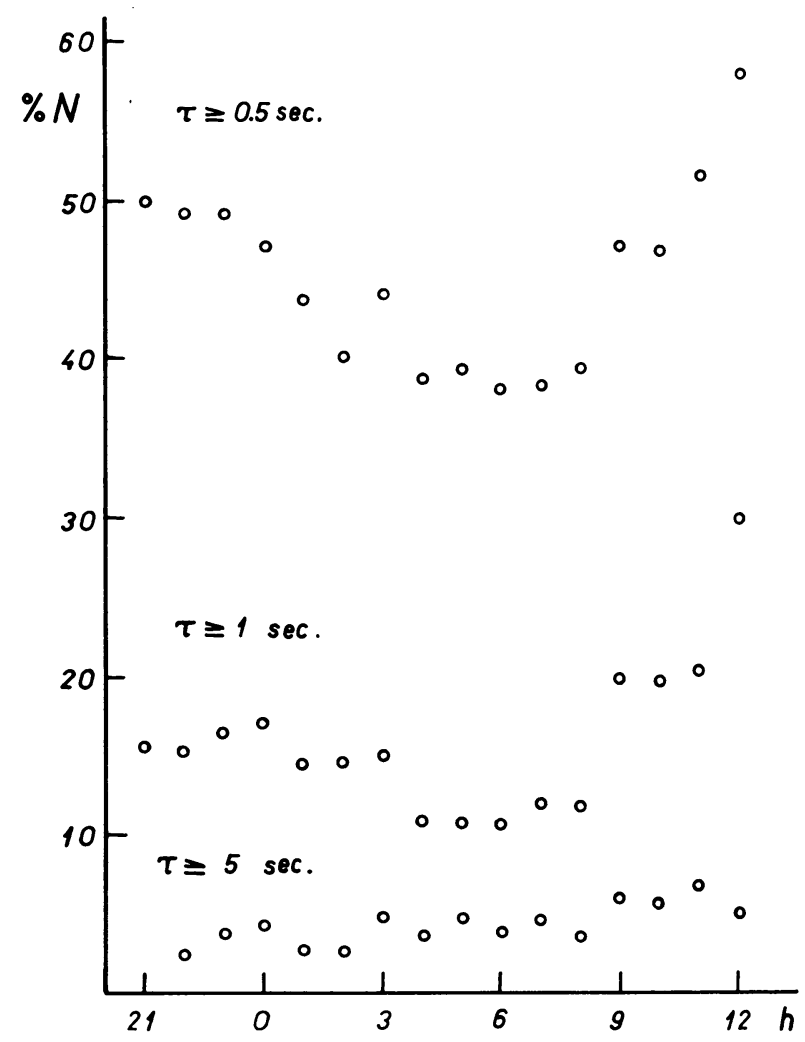

FIG. 2. The dependence of the relative number of long-duration echoes on the radiant zenith distance (Orionids 1961). Culmination at $5^{\text {h }} 20$. 
It is very interesting that the dependence constructed for Geminids is quite opposite. Figure 3 shows the relative number of echoes during Geminid period 1959 for $\tau \geqslant 0.6$ $\sec$ and $\tau \geqslant 5 \mathrm{sec}$. The symmetry to the radiant culmination is evident, but the relative number of long-duration echoes increases with increasing radiant elevation. The results for the Orionids and Geminids for the other years are very similar to those

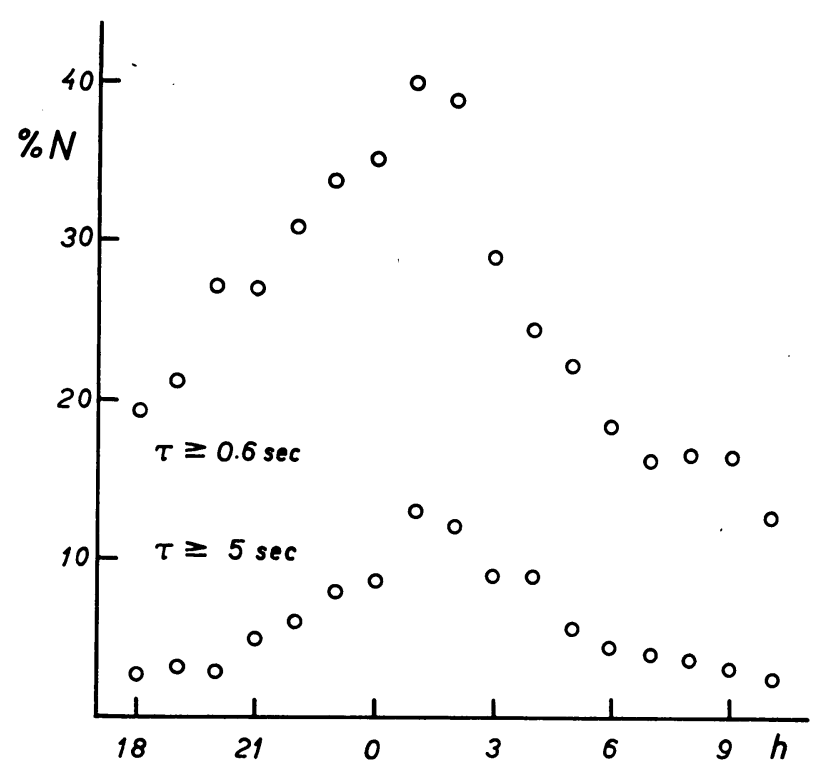

FIG. 3. The dependence of the relative number of long-duration echoes on the radiant zenith distance (Geminids 1959). Culmination at $2^{\text {h }} 00$.

in Figure 2 and Figure 3 respectively. That means that this discrepancy cannot be explained by the influence of atmospheric anomalies or other random effects.

In the analysis of the hourly range distribution of echoes, the presence of shower meteors at the characteristic ranges can be seen. The range distribution for different levels of duration, for the characteristic range with the condition of specular reflection, shows that the relative number of long-duration echoes decreases, in certain cases even more than 3 times. This decrease is equal for both showers mentioned. Only the levels of the number of echoes are different and the variation is opposite in the two cases. This effect seems to be explained by the different observational methods. While in the case of Orionids the aerial beam was fixed in azimuth at $180^{\circ}$, during the Geminid period the aerial system was changed in azimuth to $180^{\circ}$ angle from the radiant. In the two cases echoes from different areas corresponding to the different sensitivity contours were observed. (The exchange of the observational methods for these two cases would verify this result.) 
Let us look now at the magnitude ratio factor, $\kappa^{*}$, derived from the echo durations for different samples of echoes. For comparison we have the value $\kappa=3$ for the sample of all echoes for December 12-14, 1961 (ca 3000 echoes) at the time of the smallest zenith distance (01:00-04:00). In the same time, for the sample containing echoes from the range interval $230-280 \mathrm{~km}$, in which the conditions for recording shower meteors are the best (specular reflection), the value of $\kappa=3.34$ was obtained. However, for the range interval $150-200 \mathrm{~km}, \kappa=2 \cdot 24$, and for echoes with range $>300 \mathrm{~km}$, $\kappa=1 \cdot 90$. The largest difference has been obtained for the time interval 06:00-10:100 for the sample from the ranges $100-130 \mathrm{~km}$ (perpendicular to the beam) with $\kappa=5 \cdot 86$, and the sample from $155-175 \mathrm{~km}$ with $\kappa=2 \cdot 77$.

The purpose of these results is to show, that it is very difficult to draw conclusions based on the magnitude-distribution factor deduced from the radar-echo durations. Neglect of the difference of methods, time or range interval, can affect the results considerably.

\section{References}

Hajduk, A. (1965a) Bull. astr. Inst. Csl., 16, 132.

Hajduk, A. (1965b) Bull. astr. Inst. Csl., 16, 135.

Lindblad, B.A. (1956) ,Medd. Lunds astr. Obs., Ser. I, 189 and 198.

Millman, P. M., McKinley, D. W. R. (1956) Can. J. Phys., 34, 50.

\section{DISCUSSION}

Levin: What method was used to obtain the values of $\kappa$ ?

Hajduk: The magnitude-distribution factor $\kappa$ has been deduced from the number distribution of meteor-echo durations. In the first case, the value of $\kappa=3$ defines the duration interval, which is assumed constant for all samples. The corresponding ratios of the echo number yield then the values of $\kappa$ mentioned.

Whipple: Can you explain how atmospheric altitude is included in your analysis? Altitude, of course, is a major factor in determining the duration of a radar echo.

Kaiser: I would like to comment on Dr Whipple's question. One can only take account of the variation of duration with height in a statistical way, involving assumptions about the ablation process. This is one factor (dissipation of ionization by attachment, dissipation of trains by winds are others) which makes deduction of magnitude indices from duration distributions somewhat unreliable.

${ }^{*} \kappa=\frac{N_{m+1}}{N_{m}}$, where $N_{m}$ is number of meteors with magnitude $m$. 